\title{
Oncology: Future trends
}

\author{
Manoj Pandey $\cdot$ Rajan Badwe
}

The last century saw a sea change in diagnosis and treatment. These were influenced by discovery of X-rays, radium, blood groups, antibiotics, anaesthesia and exfoliative cytological techniques. These coupled with discoveries in the electronic, communication and computing has changed the face of medicine. Discovery of DNA by Watson and Crick was the most important discovery by far; which has helped in not only understanding the biological basis of cancer but has also helped in waging a war on cancer by adding another treatment modality "targeted therapy" to the existing armamentarium of treating oncologists. The coming years will see oncologist capitalising on these past discoveries and the newer ones to fight the battle with cancer. The specific issues, which will be targeted in the coming time are discussed here.

\section{Screening}

Effective screening programmes existed for cancer of the breast and cervix. Literature is fast expanding in the field of screening and over last decade screening for lung cancer, oral cancer, prostate cancer and ovarian cancer has been standardised. The screening is carried out religiously in developed countries; however, in developing countries like India, even though the concept exists on paper, it is barely implemented. The attitude of treating physicians has however, started to change and coming days will see more and more people understanding the importance of screening and will implement it. Countries like Japan has shown what screening can do. By use of routine endoscopy the incidence of oesophagus and stomach cancer has been brought down, alongwith down staging of the disease which has led to

\section{Pandey ${ }^{1} \cdot$ R. Badwe 2}

${ }^{1}$ Department of Surgical Oncology,

Institute of Medical Sciences,

Banaras Hindu University, Varanasi, UP, India

${ }^{2}$ Director and Chief of Breast Services,

Tata Memorial Hospital, Mumbai, India

M. Pandey $(\bowtie)$

E-mail: manojpandey@vsnl.com improved survival. World over there is great interest in starting the screening for other cancers and the screening programmes are still in evolution.

\section{Aetiology}

What causes cancer has always baffled researches. Enormous efforts by scientific community have led to identification of same of the genetic and biological factors that causes cancer. The stage shifted from "curse of god" to "chemical carcinogenesis' to 'genetic changes' to "gene-environment interaction" at present. For almost all cancers the genetic and molecular defects has been identified. These will help scientists tailor the treatment for each individual defect, opening the doors for gene therapy and cancer vaccines (targeted therapies).

\section{Prevention}

Identification of other aetiological factors like smoking and tobacco chewing, sunlight exposure, sexual practices, viral infections and association of diet and cancers have opened the portals for cancer prevention. Majority of these factors are avoidable hence primary cancer prevention efforts are on to prevent exposure. Anti-tobacco lobbies, prevent sun exposure campaigns are on and in future will gain in strength. It is presumed that these efforts will bring down the cancer incidence by almost $50 \%$. Identification of molecular targets has led to development of molecular prevention programmes, which are still in their infancy. Vaccine is now available for human papilloma virus and its efficacy in preventing and reducing the cervical cancer burden has already been defined, more such vaccines against bacterial or viral agents are in the pipeline.

\section{Evidence-based medicine}

The treatment in the beginning of last century was based on personal experience (read past experience) of treating physicians or their peers. This practice has fast changed 
to what is called as evidence-based medicine. More and more emphasis is being placed on conducting clinical trials. It has become mandatory to conduct clinical trials and to list them in clinical trial registers before a treatment can be accepted. The trials are conducted in stepladder fashion with first step testing for efficacy and toxicity, second for specific efficacy and third the randomised controlled trials (RCTs) to compare the new modality with established ones.

The best examples of these are trials comparing breast conservation with mastectomy, neoadjuvant chemotherapy, Herceptin $^{\circledR}$ as adjuvant, aromatase inhibitors in breast cancer, etc. At present no treatment option is acceptable without the evidence from prospective RCTs. However, there are situations where RCT may not provide evidence or may take a long time to do so. In such situation a metaanalysis may prove useful. Meta-analysis is carried out by using special statistical techniques comparing previously reported series and finding out what is the best treatment options. Best examples of meta-analysis are studies in breast cancer showing the effect of adjuvant chemo and hormonal therapies.

\section{Diagnosis}

Diagnosis of cancer with newer imaging modalities has been in vogue, ultrasonography, endoscopic ultrasound, computerised tomography (CT) scanning, magnetic resonance imaging (MRI), positron emission tomography (PET) scanning, ELISA, flow cytometry, gene fingerprinting and proteomics are examples of this.

The future will see further development of these modalities and refinement in technology for others like fusion imaging, molecular essays like western and southern blotting and gene clip technologies. It is estimated that with these developments it will be possible to identify the cancers early, even before any morphological alternation has taken place and treatment at that stage will improve the survival. A protein chip for ovarian and breast cancer and gene chip for prostate cancer is currently under clinical testing.

\section{Treatment}

Treatment of cancer in the last decade of 20th century underwent a sea change. This was seen in all established form of treatment, i.e. surgery, radiotherapy, chemotherapy, hormonal therapy and immunotherapy. Gene therapy was added to the armamentarium of treating surgeons with development of B-cell specific antibodies for lymphoma, HER-2-neu antibodies for breast cancer, anti-EGFR and anti-VEGF strategies, etc.

\section{Surgical oncology}

The century saw the surgical oncologists move from radical Halstead type of surgeries to less radical but oncologically safe surgeries, thus decreasing the morbidity of cancer surgery. Organ preservation will continue to be the key word in the coming time as well with extent of surgeries further getting reduced. The stage has shifted from surgery being the only treatment of cancer to multimodality treatment. This has led to breast conservation, limb preservation and organ preservation (especially larynx) being possible.

Technology too has come to aid of surgeons with development of staplers, hand held gamma probes, cavitron ultrasonic aspirator (CUSA), and harmonics and ultrasonics, making it possible to avoid colostomy, ileostomy, wet colostomy, debilitating surgeries, etc. It also speeded up the surgery and helped to reduce the blood loss. Future developments will see surgeons becoming more conservative and organ preservation will be the key word for all cancer at all sites.

Another development fast taking place in surgical oncology is introduction of minimal invasive surgery and robotic surgery. Non-inferiority trials have been done and the minimal access approach has been found to be oncologically safe. Introduction of robotics will further improve the results in oncology and it is already being used in prostatic and gynaeco oncology practice.

Developments are also taking place in guided surgery, use of isotopes to identify sentinel lymph nodes in various cancers has been standardised and it is expected to decrease the morbidity of lymphatic dissection. Attempts are being made at probe directed surgery using fluorescent tagged or radiolabeled monoclonal antibodies that will guide the surgeon precisely to an area with molecular alterations even if no tumour is seen with naked eye.

Developments are also taking place in other modalities like photodynamic therapy, cryo ablation, radiofrequency ablation and high intensity focal ultrasound therapy, etc. These therapies will add to the surgeons' armamentarium and he will have more options to offer then just the surgical knife.

\section{Radiotherapy}

Discovery of radium and X-rays in last century were the key to development of radiotherapy. Starting with ortho voltage radiotherapy and cobalt 60 the radiotherapy saw a change with development of linear accelerator, brachy therapy and now low, medium- and high-energy electron therapy (particle therapy) is possible without much side-effects. The future refinement in 3D radiotherapy delivering 
technique will avoid side-effects of RT. Intensity modulated radiotherapy (IMRT) and image guided radiotherapy (IGRT) are now available. Use of these techniques leads to more precise tumour kill and spares normal tissues, thus minimising the complications of radiotherapy and leads to an improved quality-of-life. Stereotactic radiotherapy for brain tumours and other vital organs is now available in select centres in India and further refinements are expected.

\section{Chemotherapy}

Chemotherapy for cancer was developed in last century and has seen multitude of changes with discovery of many chemotherapeutic agents. Last decade has seen development of compounds like colony stimulating factors for reducing chemotherapy toxicity. Further refinement in chemotherapy will see development of compounds with least toxicity to normal cells, development of compound to reduce chemotherapy toxicity and stem cell rescue, tailoring of drug delivery systems and genetically delivered drugs. Development in some of these fronts are already underway.

\section{Health education}

Probably the most important aspect of cancer management is awareness and health education. Last decade saw a change in attitude of people, awareness campaigns and a great effort to educate people. With increasing literacy rates, availability of internet and shrinkage of distance has led to increase in knowledge of people and change in their attitudes. These will be stretched in this century due to development in information technology and concentrated efforts of physicians to educate the masses. Most of the information today is available on a mouse click the only problem is the need to identify the authentic knowledge from gibberish.

\section{Conclusion}

Oncology has made tremendous progress in last century and specifically in the last decade. The developments of the last decade are being further strengthened. However, the most hope is from the developments in gene therapy, molecular markers and cancer vaccines beside health education and cancer prevention. The day is not for when we will succeed in "stopping cancer in its trek". 